\title{
On the characteristics of ASCAT wind direction ambiguities
}

\author{
W. Lin ${ }^{1}$, M. Portabella ${ }^{1}$, A. Stoffelen ${ }^{2}$, and A. Verhoef ${ }^{2}$ \\ ${ }^{1}$ Institut de Ciències del Mar (ICM) - CSIC, Barcelona, Spain \\ ${ }^{2}$ Royal Netherlands Meteorological Institute (KNMI), De Bilt, the Netherlands \\ Correspondence to: W. Lin (wenminglin@cmima.csic.es)
}

Received: 23 October 2012 - Published in Atmos. Meas. Tech. Discuss.: 18 December 2012

Revised: 1 March 2013 - Accepted: 3 April 2013 - Published: 22 April 2013

\begin{abstract}
The inversion of the Advanced Scatterometer (ASCAT) backscatter measurement triplets generally leads to two wind ambiguities with similar wind speed values and opposite wind directions. However, for up-, down- and crosswind (with respect to the mid-beam azimuth direction) cases, the inversion often leads to three or four wind solutions. In most of such cases, the inversion residuals or maximum likelihood estimators (MLEs) of the third and fourth solutions (i.e. high-rank solutions) are substantially higher than those of the first two (low-rank) ambiguities. This indicates a low probability for the high-rank solutions and thus essentially dual ambiguity. This paper investigates the characteristics of ASCAT high-rank wind solutions under different conditions with the objective of developing a method for rejecting the spurious high-rank solutions. The implementation of this rejection procedure improves the effectiveness of the ASCAT wind quality control (QC) and ambiguity removal procedures.
\end{abstract}

\section{Introduction}

The Advanced Scatterometers (ASCATs) onboard the MetOp satellite series are designed to determine the nearsurface winds over the ocean. The first ASCAT onboard MetOp-A satellite, the so-called ASCAT-A, was launched on 19 October 2006. The second onboard MetOp-B satellite, i.e. ASCAT-B, was launched on 17 September 2012. The Ocean and Sea Ice Satellite Application Facility (OSI SAF) ASCAT-A derived wind products have been operational since February 2007, whereas the OSI SAF ASCAT-B wind products are currently in development status. ASCAT operates at a microwave frequency of $5.255 \mathrm{GHz}$ ( $\mathrm{C}$ band), with three vertically polarized fan beams tracing a swath each side of the sub-satellite track (Figa-Saldana et al., 2002). In this paper the wind vector cells (WVCs) are numbered from outer swath to inner swath for both left and right swaths. For instance, WVC number 1 corresponds to the most outer-swath WVC with highest incidence angle, and WVC number 41 corresponds to the most inner-swath WVC with lowest incidence angle for $12.5 \mathrm{~km}$ ASCAT product. An important tool for interpreting data is the visualization of the three normalized radar cross section (NRCS or $\sigma^{\circ}$ ) measurements (named triplet) that correspond to the three antenna beams in 3-dimensional measurement space at each cross-track WVC (Stoffelen and Anderson, 1997). For a given WVC number, the backscatter signal mainly depends on the ocean surface wind speed and wind direction, since the parameters of geometrical measurement are fixed. In the 3-D space visualization, ASCAT-measured triplets are distributed around a welldefined "conical" surface. The latter surface is described by the forward model or geophysical model function (GMF), which has been empirically derived (Hersbach et al., 2007) as the best fit of measured backscatter to $10 \mathrm{~m}$ equivalent neutral wind vectors (Verhoef et al., 2008). The GMF relates the backscatter measurements to the observing geometry and the mean wind vector in a WVC. For a given measurement backscatter triplet in a WVC, the retrieval process consists of finding the wind speed and direction corresponding to the GMF backscatter triplet closest to the measurement. The radar antenna geometry, the measurement noise, and non-linearities in the GMF complicate the wind retrieval process, which in general leads to several solutions with similar values of the wind speed but ambiguous wind directions. These ambiguities are generally ranked by their probability or distance from the measured triplet to the GMF surface, known as the inversion residual or maximum likelihood estimator (MLE) (Stoffelen and Portabella, 2006). A spatial 
filter, the so-called ambiguity removal (AR) scheme (Stiles et al., 2002; Portabella and Stoffelen, 2004; Vogelzang et al., 2009), is then applied to produce the final or "selected" wind field. In particular, the Numerical Weather Prediction (NWP) SAF ASCAT Wind Data Processor (AWDP) uses the so-called 2D-Var AR (Vogelzang et al., 2009), an AR technique based on variational data assimilation. In contrast to other AR techniques, 2D-Var better exploits the inversion information content by explicitly using the MLE-based probability values of each wind ambiguity to retrieve the selected wind field.

Figure 1a shows the distribution of triplets (points) around a cone cross section (double ellipse), which corresponds to ASCAT WVC number 1, i.e. the near-swath WVC with lowest incidence angle. Figure $1 \mathrm{~b}$ is the same as Fig. 1a but for WVC 41, i.e. the outer-swath WVC with highest incidence angles. Note that the cross section corresponds to a roughly constant wind speed (e.g. $8 \mathrm{~m} \mathrm{~s}^{-1}$ in Fig. 1), whereas the wind direction varies along the double ellipse, such that the uppermost triplets correspond to winds blowing along the ASCAT mid-beam direction (upwind/downwind or $0^{\circ} / 180^{\circ}$ ), whereas at the lowest points the wind blows roughly across the mid-beam direction (crosswind or $90^{\circ} / 270^{\circ}$ ). The wind inversion minimizes the distance between the measured triplet and the cone surface. For triplets lying close to the cone surface, the inversion generally leads to two wind solutions or ambiguities $180^{\circ}$ apart; i.e. two specific locations on the cone surface minimize the distance due to the doubleellipse shape of the GMF. Ambiguity removal is generally not difficult in such cases (Vogelzang et al., 2008). In contrast, triplets close to the cone centre (and therefore far from the cone surface) generally lead to three-four wind solutions (Portabella et al., 2012a). Such triplets are generally affected by geophysical conditions other than those modelled by the GMF, such as rain, sea ice, confused sea state and local wind variability, thus leading to lower quality wind retrievals. A quality control (QC) scheme is used to detect and filter cases that lead to poor-quality retrievals (Stoffelen and Anderson, 1997; Portabella et al., 2012a).

Recently, we have found that near the up-, down- and crosswind directions, there are also a substantial number of triplets which lie close to cone, but have more than two solutions (see Fig. 1). Besides the first two wind solutions, which correspond to the typical dual ambiguities derived from triplets near the cone surface, there is a third and, in some cases, fourth solution typically in between the first and second solution $\left(\right.$ at $90^{\circ}$ ). A $90^{\circ}$ shift in wind direction corresponds on the cone surface to an opposing point, i.e. from up- or downwind to crosswind or vice versa. According to inversion theory, measured triplets close to the solution surface lead to good-quality wind retrievals. However, the relevance of the additional third and fourth wind solutions on the opposing side of the cone has never been assessed. That is, are these so-called "high-rank" solutions meaningful in terms of
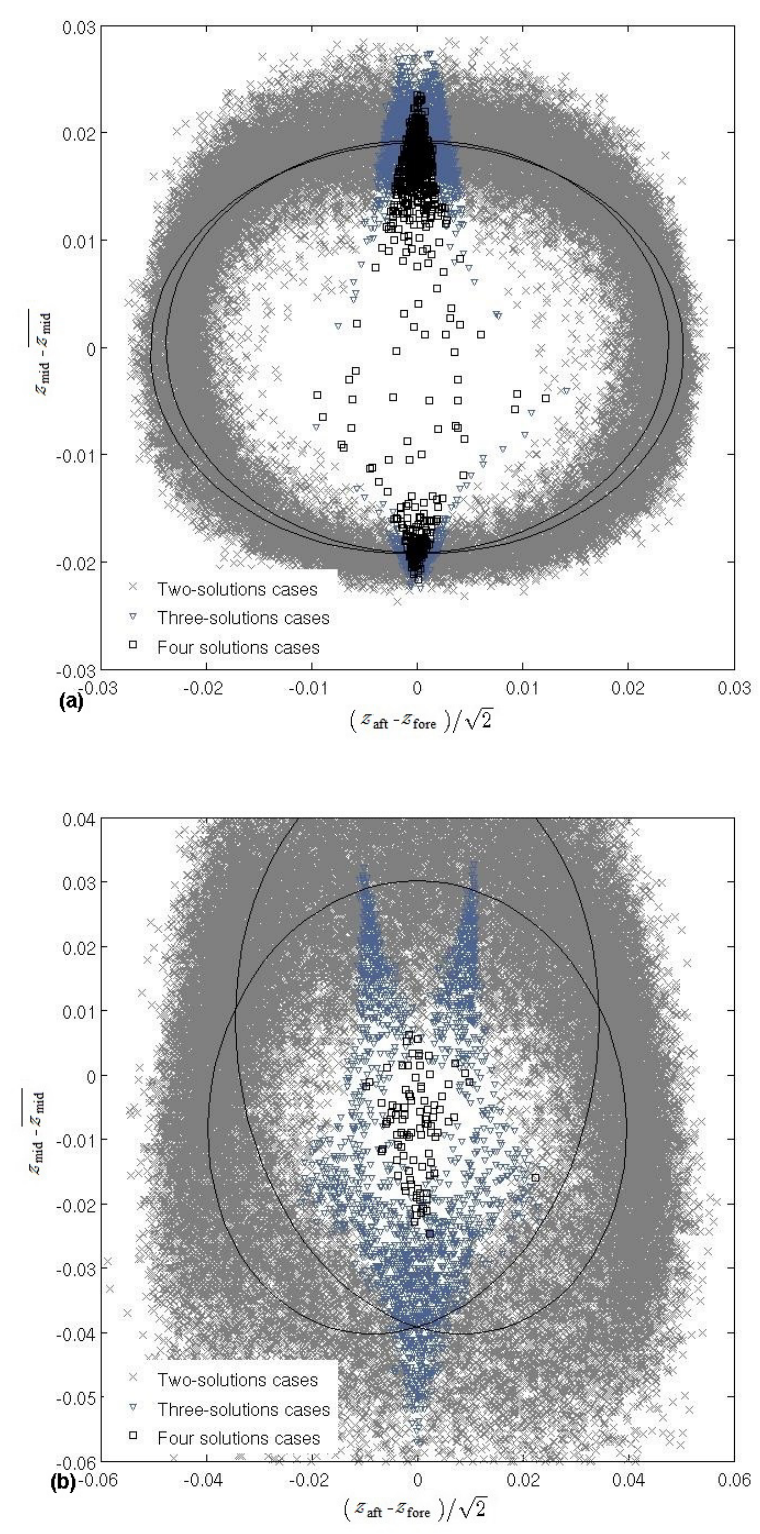

Fig. 1. Intersection of the cone with plane $z_{\text {fore }}+z_{\text {aft }}=2 z_{\text {ref }}$ for (a) WVC number 1 and (b) WVC number 41, for a value of $z_{\text {ref }}$ corresponding approximately to a speed of $8 \mathrm{~m} \mathrm{~s}^{-1}$. $z_{\text {fore }}$ and $z_{\text {aft }}$ represent the fore- and aft-beam backscatter measurements transformation in $\mathrm{z}$-space (see Eq. 2). Triplets within a distance of $\pm 0.01 z_{\text {ref }}$ from the mentioned plane are plotted. The cross, triangular and square markers in different greyscale represent the triplets with 2, 3 and 4 wind solutions respectively.

probability of being the true wind or rather artefacts of the inversion procedure?

In Sect. 2, it is shown that some "high-rank" solutions are in fact spurious and should therefore be removed after inversion (before the ambiguity removal step). In Sect. 3, a method to distinguish between the "spurious" high-rank solutions 
and the more credible high-rank solutions is proposed. Validation of this method is presented in Sect. 4. Finally, conclusions and recommendations can be found in Sect. 5.

\section{Scatterometer inversion}

Currently, the operational C-band GMF is CMOD5n (Hersbach et al., 2007; Verhoef et al., 2008), which is depicted in a transformed space, namely z-space (Stoffelen and Anderson, 1997), as follows:

$$
\begin{aligned}
z_{\mathrm{s}}(\theta, v, \phi) & =B_{0}(\theta, v)^{0.625} \times\left[1+B_{1}(\theta, v) \cos (\phi)\right. \\
& \left.+B_{2}(\theta, v) \cos (2 \phi)\right],
\end{aligned}
$$

where $\theta$ is the scatterometer incidence angle, $v$ and $\phi$ are the ocean surface wind speed and wind direction with respect to radar beam azimuth respectively. $B_{0}$ is the dominant term setting the wind speed scale, while $B_{1}$ and $B_{2}$ serve to resolve the wind direction. The particular values of $B_{0}$, $B_{1}$ and $B_{2}$ are presented in Verhoef et al. (2008). The most common approach used for scatterometer wind inversion is the maximum likelihood estimator (Cornford et al., 2004; Pierson, 1989; Stoffelen and Anderson, 1997; Stoffelen and Portabella, 2006). For ASCAT, the following MLE function is minimized (Stoffelen and Anderson, 1997):

$\operatorname{MLE}=\frac{1}{3} \sum_{i=1}^{3}\left(z_{\mathrm{m} i}-z_{\mathrm{si}}\right)^{2}$,

where $z_{\mathrm{m} i}=\left(\sigma_{\mathrm{m} i}^{\circ}\right)^{0.625}$ is the backscatter measurement of the $i$ th beam in $\mathrm{z}$-space, and $z_{\mathrm{s} i}=\left(\sigma_{\mathrm{s} i}^{\circ}\right)^{0.625}$ is the backscatter simulated with the GMF for a range of possible wind speed and direction combinations, through Eq. (1). The MLE thus facilitates the objective search in wind speed and direction for the minimum distance in a transformed (optimized) 3$\mathrm{D}$ measurement space between the measured triplet and the GMF cone surface (as illustrated in Fig. 1). The wind speed and direction solutions associated with such minima are then sorted by the MLE value; i.e. the first ranked solution corresponds to the lowest MLE value (i.e. shortest distance between the triplet and the cone surface), and so on. Note that the lower the MLE, the higher the probability is of the wind solution to be the true wind.

The MLE value is a good indicator of the retrieved wind quality (Portabella and Stoffelen, 2002). To improve the ASCAT MLE-based QC, an MLE sign has been defined by Portabella (2012a) and implemented in AWDP. The sign works as follows: triplets located inside the cone are assigned with a positive MLE value, while those located outside the cone are assigned with a negative MLE value. Note that since the cone surface has two manifolds (as represented by the double-ellipse cross section in Fig. 1), the first- and secondrank ambiguities for a triplet located between the manifolds will have opposite MLE signs; i.e. the triplet will be considered inside (outside) the cone surface for the wind solution lying on the outer (inner) cone manifold.

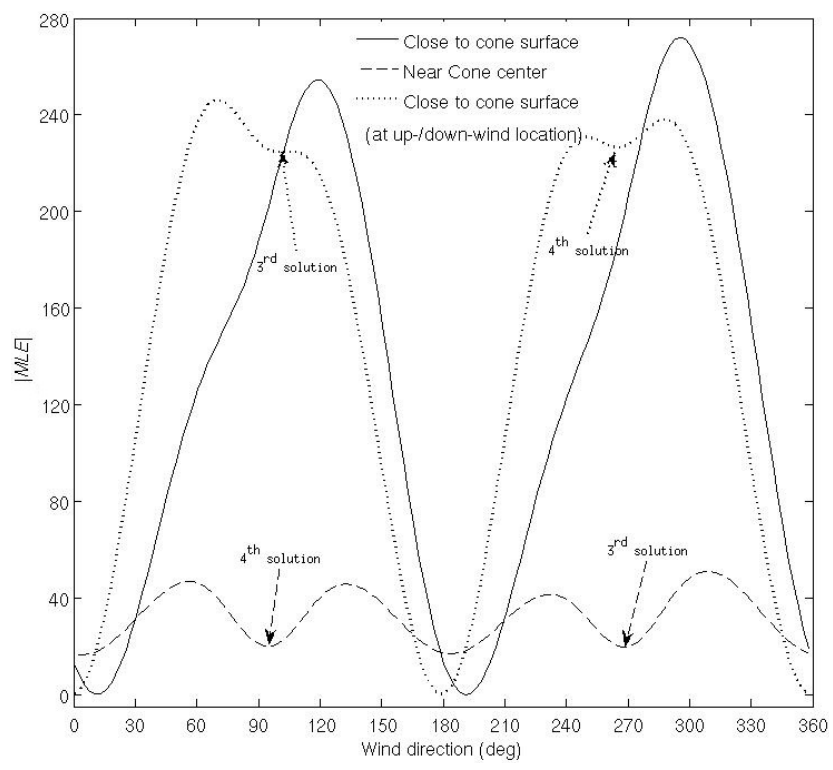

Fig. 2. Illustration of the $|\mathrm{MLE}|$ versus wind direction during the wind retrieval for three typical cases: triplet close to the cone surface (solid line), triplet near cone centre (dashed line), and triplet close to the cone surface at up-/downwind location.

As discussed in Sect. 1, when the triplets lie close to the cone surface, the inversion typically leads to two wind solutions. The solid line in Fig. 2 illustrates the MLE versus wind direction for one of such cases, where two welldefined minima have similarly low MLE values, i.e. equally and highly probable solutions. Such triplets generally lead to high-quality winds after AR.

When triplets lie far away from the cone surface (e.g. triplets located near the centre of the cross section in Fig. 1), the inversion leads to typically three or four solutions (Portabella et al., 2012a) with similar and larger MLE values (as illustrated by the dashed line in Fig. 2), i.e. up to 4 equally likely wind ambiguities. Moreover, for such cases, the minima are less well defined, as indicated by the low wind direction modulation of the dashed curve in Fig. 2 and thus have low quality. This is an indication of enhanced isotropy of ocean backscatter conditions, i.e. reduced wind direction skill, which explains the poor-quality wind vector retrieval (Stoffelen and Anderson, 1997).

The dotted line in Fig. 2 represents the wind retrieval for a triplet close to the cone surface at an up-/downwind location. There are two well-defined minima and two secondary minima. The former (first and second ranked solutions) correspond to high-probability (low MLE value) wind solutions at up-/downwind directions, and the latter (third and fourth ranked or high-rank solutions) correspond to lowprobability (high MLE value) crosswind solutions. A similar effect occurs with triplets close the crosswind direction. In this case, the well-defined minima (first- and second-rank solutions) correspond to crosswind and the secondary minima 
(high-rank solutions) to up-/downwind solutions (not shown in Fig. 2).

According to the shape of the MLE cost function curves in Fig. 2, one can clearly discern two types of distinct behaviour: triplets close to the cone (solid and dotted curves) and triplets far away from the cone (dashed curve). The former, with well-defined minima, produce high-quality winds, whereas the latter, with ill-defined minima, represent lower quality winds, some of which may be rejected. For triplets close to the cone surface at up-/downwind and crosswind locations (dotted line), the secondary minima (high-rank solutions) are poorly defined (broad) and of very low probability compared with the primary (first- and second-rank) minima (see large MLE difference between primary and secondary minima in dotted curve). They are actually produced by the particular 3-D cone shape near the measured triplet, which is driven by the GMF sensitivity to wind changes and the ASCAT observing geometry. In other words, it seems that such high-rank solutions are spurious (or meaningless) and should therefore be rejected before the AR step. According to Fig. 2, discriminating between the solid/dotted and dashed curves may be straightforward. However, in Fig. 2, two very distinct types of triplets, i.e. a triplet very close to the cone surface (solid/dotted) and a triplet very close to the centre of the cone (dashed), are represented. However, as shown in Fig. 1, a triplet lying in between the surface and the centre of the cone may not show such a distinct MLE cost function behaviour. A method to separate high-rank solutions in case of enhanced ocean isotropy (i.e. triplets close to the cone centre) from those with nominal anisotropy (i.e. triplets close to the cone surface at up-/down- and crosswind locations) is therefore required.

\section{Criteria for rejecting high-rank solutions}

To discern the characteristics of ASCAT high-rank ambiguities, $3.5 \mathrm{yr}$ (September 2008-February 2012) of OSI SAF $12.5 \mathrm{~km}$ ASCAT level 2 (L2) wind data are firstly collocated with the Tropical Rainfall Measuring Mission's (TRMM) Microwave Imager (TMI) rain data. The collocation criteria for TMI rain data are less than $30 \mathrm{~min}$ time and $0.25^{\circ}$ spatial distance from the ASCAT measurements. European Centre for Medium-range Weather Forecasts (ECMWF) winds are also used in the following analysis, which are already collected in the ASCAT L2 binary universal format representation (BUFR) data. Furthermore, a data set with buoy measurements is examined. This data set collocates $3 \mathrm{yr}$ (March 2009-February 2012) of OSI SAF $12.5 \mathrm{~km}$ ASCAT L2 BUFR data with the wind and precipitation data measured by the tropical moored buoys. The studied buoy data are provided by the National Oceanic Atmospheric Administration (NOAA) Tropical Atmosphere Ocean (TAO) buoy arrays in the tropical Pacific, the Prediction and Research Moored Array in the Atlantic (PIRATA), and the Research Moored
Array for African-Asian-Australian Monsoon Analysis and Prediction (RAMA) located in the tropical Indian Ocean.

As an initial criterion for the high-rank solution rejection procedure, no rejections are performed for wind retrievals below $4 \mathrm{~m} \mathrm{~s}^{-1}$. In contrast, above $4 \mathrm{~m} \mathrm{~s}^{-1}$ rejections are always performed for triplets lying outside the cone, i.e. when the MLE of the first and/or second rank are negative (see Sect. 2). At low wind speed conditions, ASCATs (and scatterometers in general) have poor wind direction skill (low anisotropy), i.e. low $\sigma^{\circ}$ anisotropy or wind direction modulation (Stoffelen and Anderson, 1997), and thus no dual-ambiguity highquality wind direction solutions are expected. On the other hand, for triplets lying outside the cone, which correspond to good anisotropic backscatter measurements, the retrieved winds are of high quality, as shown by Portabella (2012a), and should therefore correspond to dual ambiguity cases.

The most challenging part of the algorithm is to discriminate the high-rank solutions in backscatter conditions with enhanced isotropy from conditions with nominal anisotropy, in particular for triplets inside the cone. The MLE value can be used for such a purpose. As discussed earlier in association with Fig. 2, all solutions for triplets near the central axis of the cone have about the same distance from the GMF surface. As triplets reside closer to the cone surface, the difference in distance (MLE) between the high-rank solutions and the low-rank (first and second rank) solutions increases. Figure 3 shows the ratio between the third-ranked and the firstranked MLE (i.e. $\left|\mathrm{MLE}_{3} / \mathrm{MLE}_{1}\right|$ ) for the triplets with more than two solutions in Fig. 1, using the same vertical axis. The cross markers indicate that one of the first two ranked MLE values is negative, while the dot markers present the results for triplets with positive $\mathrm{MLE}_{1}$ and $\mathrm{MLE}_{2}$ (i.e. triplets inside the cone). Note the clear discrimination of triplets with respect to cone position, which will be further exploited here. A threshold $T$ is set to reject the meaningless high-rank solutions for those wind retrievals with positive $\mathrm{MLE}_{1}$ and $\mathrm{MLE}_{2}$. In summary, the high-rank solutions are rejected for wind retrievals with first-ranked wind speed $>4 \mathrm{~m} \mathrm{~s}^{-1}$ for all WVCs, according to the below criterion:

$\mathrm{MLE}_{1}<0$ or $\mathrm{MLE}_{2}<0$ or $\left|\mathrm{MLE}_{3} / \mathrm{MLE}_{1}\right|>T$.

Figure 1 shows that most cases with only two solutions reside near the cone surface. Triplets with high-rank solutions in case of nominal anisotropy, i.e. those triplets at up/downwind and crosswind locations, also reside near the cone surface. Therefore, the rank-1 MLE distributions of these two categories are expected to be similar. The threshold $T$ is determined by using this constraint. Figure 4 shows the MLE probability distribution function (PDF) of the first-rank solutions for two-solution cases at WVC number 1 , and a comparison to that of high-rank rejected cases for various thresholds (see legend). The standard deviation between the rank1 PDF of two-solution cases and those cases with rejected high-rank solutions is presented in the upper-left corner of Fig. 4 as a function of the threshold. The minimum value, 

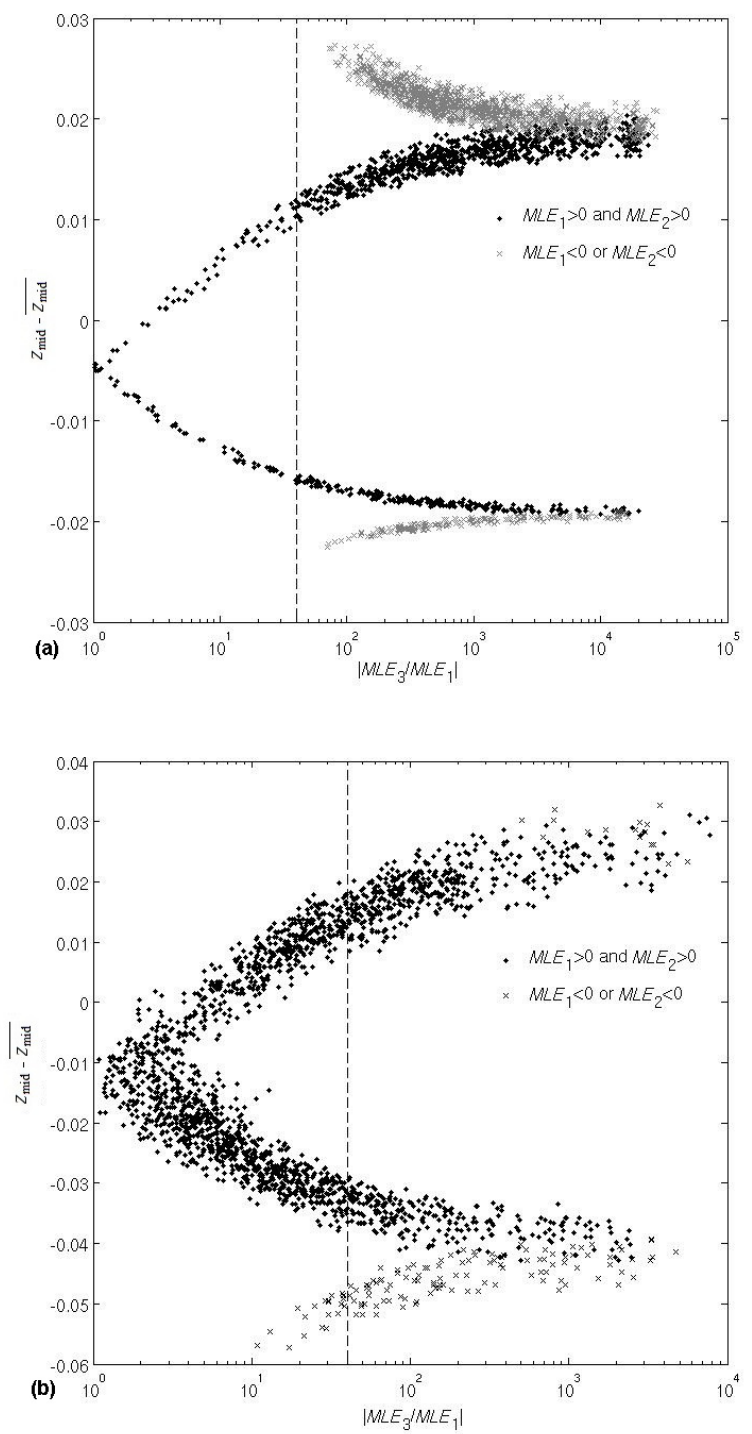

Fig. 3. (a) Illustration of the ratio $\left|\mathrm{MLE}_{3} / \mathrm{MLE}_{1}\right|$ for the triplets with more than two solutions in Fig. 1a, using the same vertical axis; (b) the same as Fig. 3a, but for the near-swath WVC, i.e. number 41. The dashed line indicates the threshold used to separate triplets with rejected high-rank solutions (right side) from those with kept high-rank solutions (left side).

which indicates the best match between the MLE distributions of the two-solution cases and cases with rejected highrank solutions, is obtained at $T=40$ for WVC number 1 . By compromising the differences of the MLE PDFs over diverse WVCs (not shown), a threshold of $T=40$ is set for the rejection procedure.

Finally, Fig. 5a shows the mean vector root-mean-square (VRMS) difference between the ASCAT-retrieved winds and the ECMWF winds, for two-solution cases (solid line), cases with rejected (dashed line) and kept (cross-marked line)

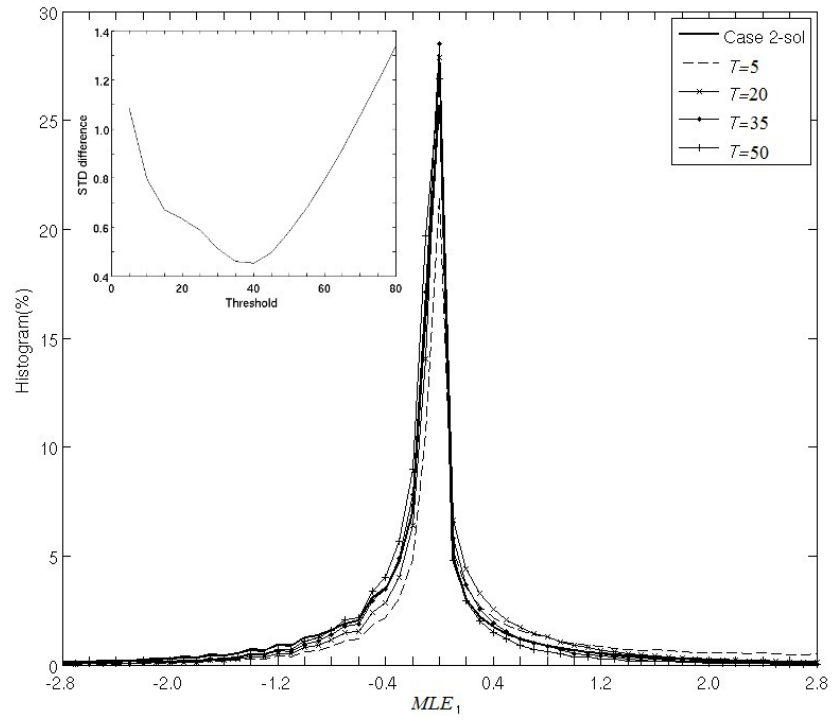

Fig. 4. Probability distribution function of the first ranked MLE at WVC number 1, for two-solution (solid line) and rejected high-rank cases with different thresholds (see legend). The standard deviation between the PDF of the two-solution cases and that of the rejected high-rank cases is illustrated as a function of the threshold in the upper left corner of this figure.

high-rank solutions. Note that only rain-free cases according to TMI collocations are taken into account. For WVCs located at outer and middle swath region (WVC number 1-30, high incidence angle), the wind retrievals with rejected highrank solutions have similar performance to that of the twosolution cases, which indicates that the proposed procedure does a good job of rejecting meaningless or geometry-related high-rank solutions. However, for inner-swath WVCs (WVC number 31-41, low incidence angle), the mean VRMS of rejected cases increases with WVC number at a higher rate than the two-solution cases, even if the threshold is enhanced to an extremely high value (not shown). In fact, this increase is mainly due to the poor rejecting performance at low wind speed (e.g. $<6 \mathrm{~m} \mathrm{~s}^{-1}$ ) conditions, in which the distribution of the ratio $\left|\mathrm{MLE}_{3} / \mathrm{MLE}_{1}\right|$ is much broader for inner-swath WVCs than for outer-swath WVCs. Regarding the wind bias and standard deviation (SD) (not shown), both the bias and the SD statistics show similar patterns for rejected and accepted high-rank solution cases at the inner-swath WVCs, indicating that the rejection procedure becomes less effective in this swath region.

Figure $5 \mathrm{~b}$ presents the same as Fig. 5a but using buoy winds instead of ECMWF as reference. Due to the lack of buoy collocations, all the collocations are examined regardless of whether they are rainy or rain-free samples. Again the mean VRMS of rejected high-rank cases is comparable with that of two-solution cases, except for the bump around WVC numbers 29-33, which is due to the very low number of collocations with rejected high-rank cases. 

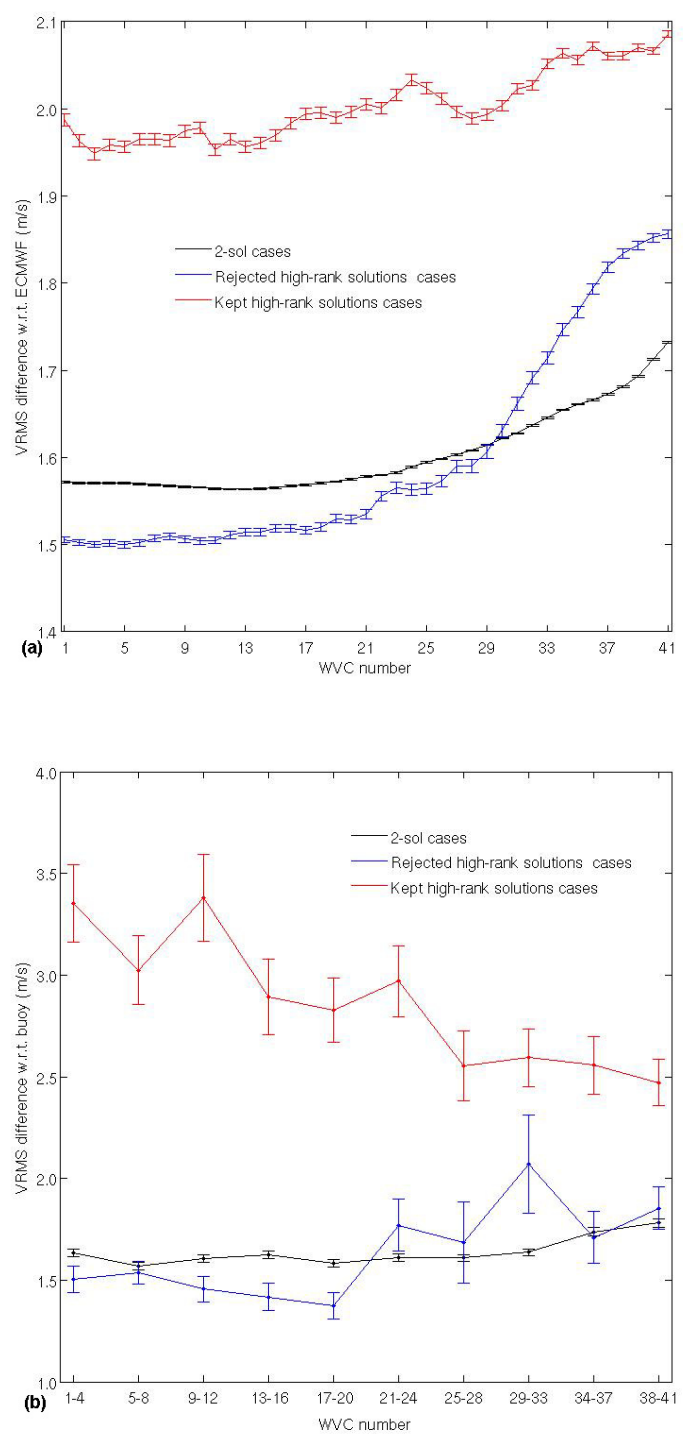

Fig. 5. The mean VRMS difference with respect to (a) ECMWF winds and (b) buoy winds as a function of WVC number, WVCs on both left and right swaths are numbered from 1 (outer-swath WVC) to 41 (near-swath WVC). Solid line indicates the result of twosolution cases; dashed line presents the result of cases with rejected high-rank solutions with threshold $T=40$, and cross-marked line illustrates the result of cases with kept high-rank solutions. Marker "I" denotes the uncertainty bar of the estimated mean VRMS for each WVC bin.

\section{Analysis of the effectiveness of rejecting high-rank solutions}

To verify the impact of the high-rank solution rejection procedure on ASCAT wind retrievals, the number of geometryrelated high-rank solutions that would be selected by the 2DVar AR module if they were not rejected is examined. This number divided by the total number of cases with rejected
Table 1. The percentage of triplets with rejected high-rank solutions that were selected by the AR module. WVC number 1 corresponds to highest incidence angle (outer-swath WVC), and WVC number 41 corresponds to lowest incidence angle (near-swath WVC).

\begin{tabular}{llrrr}
\hline \multicolumn{2}{c}{ Wind speed $\left(\mathrm{m} \mathrm{s}^{-1}\right)$} & $4<v \leq 6$ & $6<v \leq 10$ & $v>10$ \\
\hline \multirow{2}{*}{ WVC number 1 } & Rain-free & 0.3 & 0.07 & 0.07 \\
& Rainy & 5.3 & 3.6 & 3.9 \\
\hline \multirow{2}{*}{ WVC number 41 } & Rain-free & 2.2 & 0.5 & 0 \\
& Rainy & 11.2 & 6.9 & 3.2 \\
\hline
\end{tabular}

high-rank solutions is denoted by $R_{\mathrm{S}}$. Ideally, $2 \mathrm{D}$-Var AR should only select a geometry-related high-rank solution in very few cases and rather generally "stick" to either the firstor the second-rank solution. For example, assuming that the wind direction uncertainty is characterized by a Gaussian distribution, the proportion of data (wind direction) values within 45 degrees is $99.73 \%$ (or $95.45 \%$ ) provided that the 2D-Var uncertainty is 15 degrees (or 22.5 degrees). In other words, the percentage of values beyond 45 degrees is $0.26 \%$ (or $4.55 \%$ ). Therefore, if a local wind direction error of 45 degrees allows the selection of a high-rank solution, then its probability of occurrence would be approximately $0.3 \%$ in the case of a 15-degree 2D-Var uncertainty and $4.5 \%$ for 22.5-degree uncertainty. The latter uncertainty may occur for low winds, while the former is more typical for winds of nominal strength. Both TMI rain-free and rainy collocations are studied. Table 1 presents the $R_{\mathrm{S}}$ results for different WVC number and geophysical conditions. It shows that the ratio $R_{\mathrm{S}}$ decreases with increasing wind speed as expected. For innerswath WVCs, the higher $R_{\mathrm{S}}$ value indicates that it is not easy to figure out the geometry-related high-rank solutions, probably due to increased wind direction uncertainty caused by reduced GMF sensitivity for lower incidence angles.

The rejected high-rank solutions are more likely to be chosen by the AR module of L2 processing in rainy areas, as compared to the rain-free cases. Since ECMWF winds do not resolve wind variability and downdrafts in rainy areas (Portabella et al., 2012b), it is supposed that the inaccurate background winds may lead to the selection of spurious highrank solutions through the AR processing. In other words, it is important to reject the meaningless high-rank solutions, especially for rainy conditions.

The validation using buoy data is also examined. Within the total of 86000 collocations, there are 6140 cases with more than two solutions, among which 2959 are WVCs with rejected high-ranked solutions according to the procedure in Sect. 3. The $R_{\mathrm{S}}$ value for the buoy collocations is $1.1 \%$ (i.e. 33 cases with rejected high-rank solutions, but which are selected by the AR module). Furthermore, within the $R_{\mathrm{s}}$ determined category, there are 20 cases in which the first two ranked solutions are closer to the collocated buoy wind than the higher ranks. For the other 13 cases, the selected 
high-rank solutions diverge more than 30 degrees from the buoy wind direction, but are in slightly better agreement with the buoy than the first two solutions. This is an indication of potential rain-contaminated ASCAT winds. Such poorquality cases should be quality-controlled, i.e. all solutions rejected rather than only the high-rank solutions. Although rejecting high-rank solutions may lead to MLE-based QCpassed WVCs (MLE of first- and second-rank solutions is usually low for rejected high-rank cases), the latter can easily be filtered by the 2D-Var QC, which checks consistency between the ASCAT wind solutions and the background or 2D-Var analysed field.

In $16.7 \%$ of cases, 2D-Var selected a high-rank solution from the cases with kept high-rank solutions. The mean VRMS difference with the buoy winds is then relatively high and $4.45 \mathrm{~m} \mathrm{~s}^{-1}$, as compared to $2.53 \mathrm{~m} \mathrm{~s}^{-1}$ in cases where the first- or second-rank solution was selected. In $54.6 \%$ of cases, the selected high-rank solution was also the closest to the buoy.

\section{Conclusions}

In cases where the ocean return is rather isotropic, inversion of ASCAT backscatter triplets results in more than two solutions; i.e. high-rank solutions (up to four) emerge due to reduced wind direction skill (e.g. in cases of, high subWVC wind variability, rain contamination, etc.). These cases are well represented through these additional wind direction ambiguities, which need to be kept. On the other hand, for ASCAT measurement triplets located close to the GMF (cone surface), the inversion procedure results in two wind ambiguities, except for triplets located at up-, down- and crosswind locations. These additional and artificial high-rank solutions appear due to the cone geometry, which is driven by the ASCAT measurement geometry and the GMF sensitivity to wind speed and direction changes. To filter out these geometry-related high-rank solutions, an MLE-based method is proposed. The rationale is to reject these meaningless high-rank solutions and avoid the selection of "spurious" ambiguities during the quality control and ambiguity removal steps.

The third- and fourth-rank rejection criteria are the following: (a) no rejections for ASCAT winds below $4 \mathrm{~m} \mathrm{~s}^{-1}$ (since these are generally cases with poor wind direction skill); (b) for winds above $4 \mathrm{~m} \mathrm{~s}^{-1}$, reject for triplets outside the cone surface; and (c) reject when $\left|\mathrm{MLE}_{3} / \mathrm{MLE}_{1}\right|>=40$, for triplets inside the cone. It is found that the quality (using both ECMWF and buoy winds as reference) of the less ambiguous (with rejected high-rank solutions) WVCs is similar to that of the dual-ambiguity cases, whereas the quality of fully ambiguous (with kept third and fourth ranks) WVCs is much lower, as expected (since they correspond to poorquality cases). However, for inner-swath WVCs, where the wind direction skill is somewhat lower, the rejection proce- dure is less effective, suggesting that no rejections should be performed for such WVCs below $6 \mathrm{~m} \mathrm{~s}^{-1}$.

Rejected high ranks are more likely to be selected by the AR module (denoted as $R_{\mathrm{S}}$ cases) over rainy areas than over dry areas, which suggests a more negative effect of such cases in rainy conditions when not rejected. However, a significant amount of $R_{\mathrm{S}}$ cases show high-rank solutions to be (slightly) closer to buoy data than low-rank solutions. This shows a potential ASCAT rain-contamination effect on ASCAT WVCs. For such cases, a complementary QC is required since the MLE-based QC does not filter them (triplets are close to the cone surface). An alternative QC has been recently presented by Portabella (2012b) with promising preliminary results. However, further work is required to improve ASCAT rain correction and QC under rainy conditions.

When more collocations of ASCAT, buoy wind and precipitation data become available, a quantitative study of the impact high-rank solutions on both AR and QC in L2 processing will be carried out.

Acknowledgements. This work was supported in part by the European Organisation for the Exploitation of Meteorological Satellites Ocean and Sea Ice Satellite Application Facility Associated Scientist project under Reference CDOP-SG06-VS03 and in part by the Spanish Ministry of Science and Innovation (MICINN) National R\&D project under Reference AYA2010-22062-C05. The authors would like to thank J.-R. Bidlot and ECMWF for providing the GTS buoy wind data set (already quality controlled). The ASCAT level $1 \mathrm{~b}$ data are provided by EUMETSAT. The software used in this paper was developed through the EUMETSAT NWP SAF. The ECMWF data were retrieved from the ECMWF MARS archive. The TMI data are available from the Web site of Remote Sensing Systems (http://www.ssmi.com/). The authors greatly appreciate the three reviewers who helped to improve the paper.

Edited by: F. S. Marzano

\section{References}

Cornford, D., Csató, L., Evans, D. J., and Opper, M.: Bayesian analysis of the scatterometer wind retrieval inverse problems: some new approaches, J. R. Statist. Soc. B., 66, 609-626, 2004.

Figa-Saldana, J., Wilson, J. J. W., Attema, E., Gelsthorpe, R., Drinkwater, M. R., and Stoffelen, A.: The advanced scatterometer (ASCAT) on Metop: A follow-on for European scatterometers, Can. J. Remote Sens., 28, 404-412, 2002.

Hersbach, H., Stoffelen, A., and De Haan, S.: The improved C-band ocean geophysical model function: CMOD-5, J. Geophys. Res., 112, C03006, doi:10.1029/2006JC003743, 2007.

Pierson, W. J.: Probabilities and statistics for backscatter estimates obtained by a scatterometer, J. Geophys. Res., 94, 9743-9759, 1989.

Portabella, M. and Stoffelen, A.: Characterization of residual information for SeaWinds quality control, IEEE Trans. Geosci. Remote Sens., 40, 2747-2759, 2002. 
Portabella, M. and Stoffelen, A.: A probabilistic approach for SeaWinds data assimilation, Q. J. Roy. Meteorol. Soc., 130, 127152, 2004.

Portabella, M., Stoffelen, A., Verhoef, A., and Verspeek, J.: A new method for improving scatterometer wind quality control, IEEE Trans. Geosci. Remote. Sens. Lett., 9, 579-583, 2012a.

Portabella, M., Stoffelen, A., Lin, W., Turiel, A., Verhoef, A., Verspeek, J., and Ballabrera, J.: Rain effects on ASCAT wind retrieval: Towards an improved quality control, IEEE Trans. Geosci. Remote Sens., 50, 2495-2506, 2012b.

Stiles, B. W., Pollard, B. D., and Dunbar, R. S.: Direction interval retrieval with thresholded nudging: A method for improving the accuracy of QuikSCAT winds, IEEE Trans. Geosci. Remote. Sens., 40, 79-89, 2002

Stoffelen, A. and Anderson, D.: Scatterometer data interpretation: measurement space and inversion, J. Atmos. Ocean. Technol., 14, 1298-1313, 1997.

Stoffelen, A. and Portabella, M.: On Bayesian scatterometer wind inversion, IEEE Trans. Geosci. Remote. Sens., 44, 1523-1533, 2006.
Verhoef, A., Portabella, M., Stoffelen, A., and Hersbach, H.: CMOD5.n- the CMOD5 GMF for neutral winds, Ocean and Sea Ice SAF Technical Note, SAF/OSI/CDOP/KNMI/TEC/TN/ 165 v.1, May 2008, available at: http://www.knmi.nl/scatterometer/ publications/, 2008.

Vogelzang, J., Stoffelen, A., Belmonte, M., Portabella, M., Verhoef, A., and Verspeek, J.: Quality of high resolution ASCAT wind fields. Proc. 2008 EUMETSAT Meteorological satellite Conference, Darmstad, Germany, 8-12 September 2008, available at: http://www.eumetsat.int/idcplg? IdcService=GET_FILE\&dDocName=PDF_CONF_P_S3_09_ VOGELZAN_P\&RevisionSelectionMethod=LatestReleased (last access: 18 April 2013), 2008.

Vogelzang, J., Stoffelen, A., Verhoef, A., De Vries, J., and Bonekamp, H.: Validation of two-dimensional variational ambiguity removal on SeaWinds scatterometer data, J. Atmos. Ocean. Technol., 26, 1229-1245, 2009. 